\title{
Cardiovascular Effects of Stimulant and Non-Stimulant Medication for Children and Adolescents with ADHD: A Systematic Review and Meta-Analysis of Trials of Methylphenidate, Amphetamines and Atomoxetine
}

\author{
Leonie Hennissen $^{1}$ (D) Mireille J. Bakker $^{1} \cdot$ Tobias Banaschewski $^{3}$ • \\ Sara Carucci ${ }^{4}$ - David Coghill ${ }^{5,14} \cdot$ Marina Danckaerts $^{6} \cdot$ Ralf W. Dittmann $^{3}$. \\ Chris Hollis $^{7} \cdot$ Hanna Kovshoff $^{8} \cdot$ Suzanne McCarthy $^{9} \cdot$ Peter Nagy $^{10}$. \\ Edmund Sonuga-Barke $^{8}$ - Ian C. K. Wong ${ }^{12,13} \cdot$ Alessandro Zuddas ${ }^{4} \cdot$ \\ Eric Rosenthal $^{11} \cdot$ Jan K. Buitelaar ${ }^{1,2}$. The ADDUCE consortium
}

Published online: 24 February 2017

(C) The Author(s) 2017. This article is published with open access at Springerlink.com

\begin{abstract}
Background Many children and adolescents with attention deficit/hyperactivity disorder (ADHD) are treated with stimulant and non-stimulant medication. ADHD medication may be associated with cardiovascular effects. It is important to identify whether mean group effects translate into clinically relevant increases for some individual patients, and/or increase the risk for serious cardiovascular adverse events such as stroke or sudden death.
\end{abstract}

Electronic supplementary material The online version of this article (doi:10.1007/s40263-017-0410-7) contains supplementary material, which is available to authorized users.

Jan K. Buitelaar

Jan.Buitelaar@radboudumc.nl

1 Department of Cognitive Neuroscience (204), Donders Institute for Brain, Cognition and Behaviour, Radboud University Medical Center, P.O. Box 9101, 9100 HB Nijmegen, The Netherlands

2 Karakter Child and Adolescent Psychiatry University Centre, Nijmegen, The Netherlands

3 Paediatric Psychopharmacology, Department of Child and Adolescent Psychiatry, Central Institute of Mental Health, Medical Faculty Mannheim, University of Heidelberg, Mannheim, Germany

4 Child and Adolescent Neuropsychiatric Unit, Department of Biomedical Sciences, University of Cagliari and "A. Cao" Microcitemico Paediatric Hospital, Cagliari, Italy

5 Division of Neuroscience, School of Medicine, University of Dundee, Ninewells Hospital and Medical School, Dundee, UK

6 Child and Adolescent Psychiatry, Department of Neurosciences, KU Leuven, Louvain, Belgium
Objectives To evaluate potential cardiovascular effects of these treatments, we conducted a systematic review and meta-analysis of the effects of methylphenidate (MPH), amphetamines (AMP), and atomoxetine (ATX) on diastolic and systolic blood pressure (DBP, SBP) and heart rate (HR) in children and adolescents with ADHD.

Methods We conducted systematic searches in electronic databases (PsychINFO, EMBASE and Medline) to identify published trials which involved individuals who were (i) diagnosed with ADHD and were aged between 0-18 years; (ii) treated with MPH, AMP or ATX and (iii) had their DBP and SBP and/or HR measured at baseline (pre) and the endpoint (post) of the study treatment. Studies

7 Division of Psychiatry and Applied Psychology, University of Nottingham, Nottingham, UK

8 Department of Psychology, University of Southampton, Highfield, Southampton, UK

9 School of Pharmacy, University College Cork, Cork, Ireland

10 Vadaskert Child and Adolescent Psychiatric Hospital, Budapest, Hungary

11 Department of Paediatric Cardiology, Evelina London Children's Hospital, St Thomas' Hospital, London, UK

12 Research Department of Practice and Policy, UCL School of Pharmacy, 29-39 Brunswick Square, London WC1 N 1AX, UK

13 Department of Pharmacology and Pharmacy, University of Hong Kong, Hong Kong, China

14 Departments of Paediatrics and Psychiatry, Faculty of Medicine, Dentistry and Health Sciences, University of Melbourne, Parkville, Australia 
with an open-label design or a double-blind randomised control design of any duration were included. Statistical analysis involved calculating differences between pre- and post-treatment measurements for the various cardiovascular parameters divided by the pooled standard deviation. Further, we assessed the percentage of clinically relevant increased BP or HR, or documented arrhythmias.

Results Eighteen clinical trials met the inclusion criteria (10 for MPH, 5 for AMP, and 7 for ATX) with data from 5837 participants $(80.7 \%$ boys) and average duration of 28.7 weeks (range 4-96 weeks). All three medications were associated with a small, but statistically significant pre-post increase of SBP (MPH: standard mean difference [SMD] $0.25,95 \%$ confidence interval [CI] 0.08-0.42, $p<0.01$; AMP: SMD 0.09, 95\% CI 0.03-0.15, $p<0.01$; ATX: SMD 0.16, 95\% CI 0.04-0.27, $p=0.01$ ). MPH did not have a pre-post effect on DBP and HR. AMP treatment was associated with a small but statistically significant prepost increase of DBP (SMD 0.16, CI 0.03-0.29, $p=0.02$ ), as was ATX treatment (SMD 0.22, CI 0.10-0.34, $p<0.01$ ). AMP and ATX were associated with a small to medium statistically significant pre-post increase of HR (AMP: SMD 0.37, CI 0.13-0.60, $p<0.01$; ATX: SMD 0.43 , CI $0.26-0.60, p<0.01)$. The head-to-head comparison of the three medications did not reveal significant differences. Sensitivity analyses revealed that AMP studies of $<18$ weeks reported higher effect sizes on DBP compared with longer duration studies $(F(1)=19.55$, $p=0.05$ ). Further, MPH studies published before 2007 reported higher effect sizes on SBP than studies after 2007 $(F(1)=5.346, p=0.05)$. There was no effect of the following moderators: type of medication, doses, sample size, age, gender, type of ADHD, comorbidity or dropout rate. Participants on medication reported 737 (12.6\%) other cardiovascular effects. Notably, $2 \%$ of patients discontinued their medication treatment due to any cardiovascular effect. However, in the majority of patients, the cardiovascular effects resolved spontaneously, medication doses were changed or the effects were not considered clinically relevant. There were no statistically significant differences between the medication treatments in terms of the severity of cardiovascular effects.

Conclusions Statistically significant pre-post increases of SBP, DBP and HR were associated with AMP and ATX treatment in children and adolescents with ADHD, while MPH treatment had a statistically significant effect only on SBP in these patients. These increases may be clinically significant for a significant minority of individuals that experience larger increases. Since increased BP and HR in general are considered risk factors for cardiovascular morbidity and mortality during adult life, paediatric patients using ADHD medication should be monitored closely and regularly for HR and BP.

\section{Key Points}

Attention deficit/hyperactivity disorder (ADHD) is a common neuropsychiatric disorder for which medication plays a pivotal role for clinical management.

Amphetamine and atomoxetine were associated with small but statistically significant pre-post increases in systolic and diastolic blood pressure and heart rate in children and adolescents with ADHD, while methylphenidate treatment had this effect only on systolic blood pressure in these individuals.

Of the participants on medication, $12.6 \%$ reported other cardiovascular effects and $2 \%$ discontinued their medication treatment due to any cardiovascular effect; other cardiovascular effects resolved spontaneously, medication doses were changed or the effects were not considered clinically relevant. There were no significant differences in terms of the severity of cardiovascular effects between the medication treatments.

More research into the long-term effects on the left ventricular mass of these relatively small changes of blood pressure and heart rate associated with ADHD medication treatment is required.

\section{Introduction}

Attention deficit/hyperactivity disorder (ADHD) is one of the most common childhood psychiatric disorders, affecting about $3-5 \%$ of all school-age children worldwide $[1,2]$. ADHD is characterised by clinically significant levels of inattentive, and/or impulsive and hyperactive behaviours [3]. ADHD is a lifespan condition with a large proportion of paediatric patients continuing to exhibit symptoms into adolescence and adulthood [4]. Clinical guidelines and practice parameters describe the pivotal role of medication in the clinical management of ADHD [5-9]. These recommendations are based on numerous clinical trials that have shown both stimulant [e.g. methylphenidate (MPH) and amphetamine (AMP)] and non-stimulant medication [e.g. atomoxetine (ATX)] to be efficacious in treating the symptoms of ADHD, with over $70 \%$ of the reported patients being considered clinical responders [5-9]. Given the persistence of ADHD symptoms, patients with ADHD often require long-term pharmacotherapy. Of those who begin treatment for ADHD, 18-50\% continue medication for a significant period (e.g. 2-3 years [10]). 
While, in general, ADHD medications are well tolerated, safety and adverse events are the focus of significant clinical and public health concern. Adverse events like sleep and eating problems are commonly reported and can usually be managed by making changes to the medication regimen or daily routines [11]. Based on mechanism of action, there is also the potential for adverse impacts on cardiac functioning with these medications. MPH, AMP and ATX are all sympathomimetic agents that increase noradrenergic and dopaminergic transmission: an effect on heart rate (HR) and blood pressure (BP) can therefore be considered an intrinsic feature of their pharmacological activity $[12,13]$.

Whilst some authors have described the overall cardiac risks associated with ADHD medication as unimportant [14], the available evidence from individual studies suggests that MPH and ATX may be associated with generally small elevations of $\mathrm{BP}(\leq 5 \mathrm{mmHg})$ and $\mathrm{HR}(\leq 10$ beats/min $[\mathrm{bpm}])$ at a group level but no changes in electrocardiographic (ECG) parameters [15]. Similar increases of BP and HR have been reported for AMP [16]. It is, however, possible that a subset of children and adolescents (around 5-15\%) may experience greater treatment-related increases in $\mathrm{HR}$ or $\mathrm{BP}$, or may report a cardiovascular-type complaint during ADHD medication treatment [15]. A meta-analysis in adult patients with ADHD reported that treatment with stimulants is associated with small but significant increases of systolic blood pressure (SBP) $(+2.0 \mathrm{mmHg})$ and $\mathrm{HR}(+5.7 \mathrm{bpm})$ but no effect on diastolic blood pressure (DBP) [17].

It is therefore important to identify whether these cardiac effects of ADHD medication seen at the group level, translate into clinically relevant increases for some individual patients, and/or increase the risk for serious cardiovascular adverse events such as stroke or sudden death. Apart from one study [18], for which possible methodological limitations were identified [19], available epidemiological research has not shown a significant association between treatment with ADHD medication and the occurrence of serious cardiovascular events in children and adolescents [20, 21]. One retrospective study reported "an overall rate of 2.8 cardiovascular events per 100,000 patients". The authors concluded that medication treatment for children with ADHD is not significantly associated with increased risk of stroke, acute myocardial infarction, sudden cardiac death or ventricular arrhythmia [22]. A pooled analysis of three other large retrospective data sets [23-25] including more than 1.8 million MPH-, AMP- and ATX-treated patients reported no association between MPH, AMP and ATX and sudden death or stroke [26]. In contrast, a prospective longitudinal nationwide cohort study $(n=714,258)$ reported stimulant-related increased risk of cardiovascular events (adjusted hazard ratio 1.83). The effects were still present when the analysis was restricted to children with $\operatorname{ADHD}(n=8300)$ who used stimulants (adjusted hazard ratio 2.20; [27]). However, the definition of 'cardiovascular event' in this study was nonspecific, and included hypertension, rheumatic fever and "cardiovascular disease not otherwise specified", which makes it difficult to draw definitive conclusions.

\section{Methods}

\subsection{Objectives and Inclusion Criteria}

Here we report the results of the first review and metaanalysis of cardiovascular outcomes with ADHD medication in children and adolescents. We assessed effects of three types of medications used for the treatment of ADHD (MPH, AMP, ATX) on DBP, SBP and HR. We addressed the following questions:

1. Are MPH, AMP and ATX associated with an increase in DBP, SBP and HR?

2. What is the rate of clinically relevant changes of DBP, $\mathrm{SBP}$, and HR at an individual level?

3. Are these effects moderated by age, sex, comorbidity, medication variables such as dosage and type of medication, duration of treatment, dropout-rate and publication year?

We included trials published in peer-reviewed journals at any time from a database's inception. Studies were included if they met the following criteria:

1. Participants under the age of 18 years who had a diagnosis of ADHD of any subtype (according to the DSM-III, DSM-III-R or DSM-IV or of hyperkinetic disorder according to the ICD-10 system) or met accepted criteria for clinical levels of symptoms on validated ADHD rating scales.

2. MPH, AMP or ATX medication was prescribed to patients during the study (regardless of the treatment duration). Studies were only included if medications were prescribed more than once to each participant (always the same doses or up-titrated doses).

3. At least one cardiovascular outcome was reported (DBP, SBP and/or HR).

4. Both the baseline (pre) and the endpoint (post) of the treatment were available.

As there are very few randomised controlled trials that report adequate systematic data on cardiovascular outcome (DBP, SBP and HR), open-label and double-blinded study designs without a control group were included. Clonidine and guanfacine studies were not included because there were very few studies published on these medications. In particular, in Europe, guanfacine was labelled for ADHD and brought to market only very recently. 


\subsection{Search Strategy}

We conducted a systematic and comprehensive search for peer-reviewed papers from the initiation of the databases until 15 May 2015 in Medline, PsychINFO and EMBASE. Only articles including humans and written in English were included. We used common terms for participants (all variants of ADHD, hyperkinetic disorder, attention deficit), medications and adverse events across domains (see Appendix 1, electronic supplementary material). The medication terms were methylphenidate or methylphenidate hydrochloride, methylphenidate hcl, metadate, medikinet, methylin, ritalin, equasym, daytrana, concerta, atomoxetine, atomoxetin, strattera, attentrol, attentin, recit, tomoxetin, amphetamine, amfetamine, amphetamines, amfetamines, levoamphetamine, levoamfetamine, dextroamphetamine, dextroamfetamine, methamphetamine, methamfetamine, detraamphetamine, detraamfetamine, lisdexamphetamine, lisdexamfetamine, dexamphetamine, dexamfetamine, benzedrine, adderall, desoxyn, dexedrine, destrostat, vyvanse. The adverse event terms were adverse event, adverse effect or adverse effects, adverse reaction, adverse reactions, side effect, side effects, untoward effect, untoward effects, adverse drug experience, adverse drug reactions, drug experience report, drug experience reports, toxic reaction, toxic reactions, toxic effect, toxic effects, complication, complications, undesired effect, undesired effects, unwanted drug effect, unwanted drug effects. In addition, we manually searched bibliographies from identified articles and relevant published reviews to identify additional related publications. We contacted authors to gather information for required fields if they were not reported in the included papers. We excluded any case reports. Furthermore, we excluded single-dose studies and studies with only a baseline measurement for different doses (see Fig. 1 for a flow chart of the search strategy).
Fig. 1 Preferred Reporting Items for Systematic Reviews and Meta-Analyses (PRISMA ${ }^{\mathrm{a}}$ ) flow diagram on cardiovascular effects. ${ }^{\mathrm{a}}$ PRISMA Preferred Reporting Items for Systematic Reviews and Meta-Analyses (http://www.prisma-statement. org). ${ }^{b}$ References from identified articles and relevant published reviews were manually searched to identify additional related publications. ${ }^{\mathrm{c}}$ These studies were about the influence of doses on cardiovascular measurements and had no post measurement. $M P H$ methylphenidate, $A M P$ amphetamines, $A T X$ atomoxetine

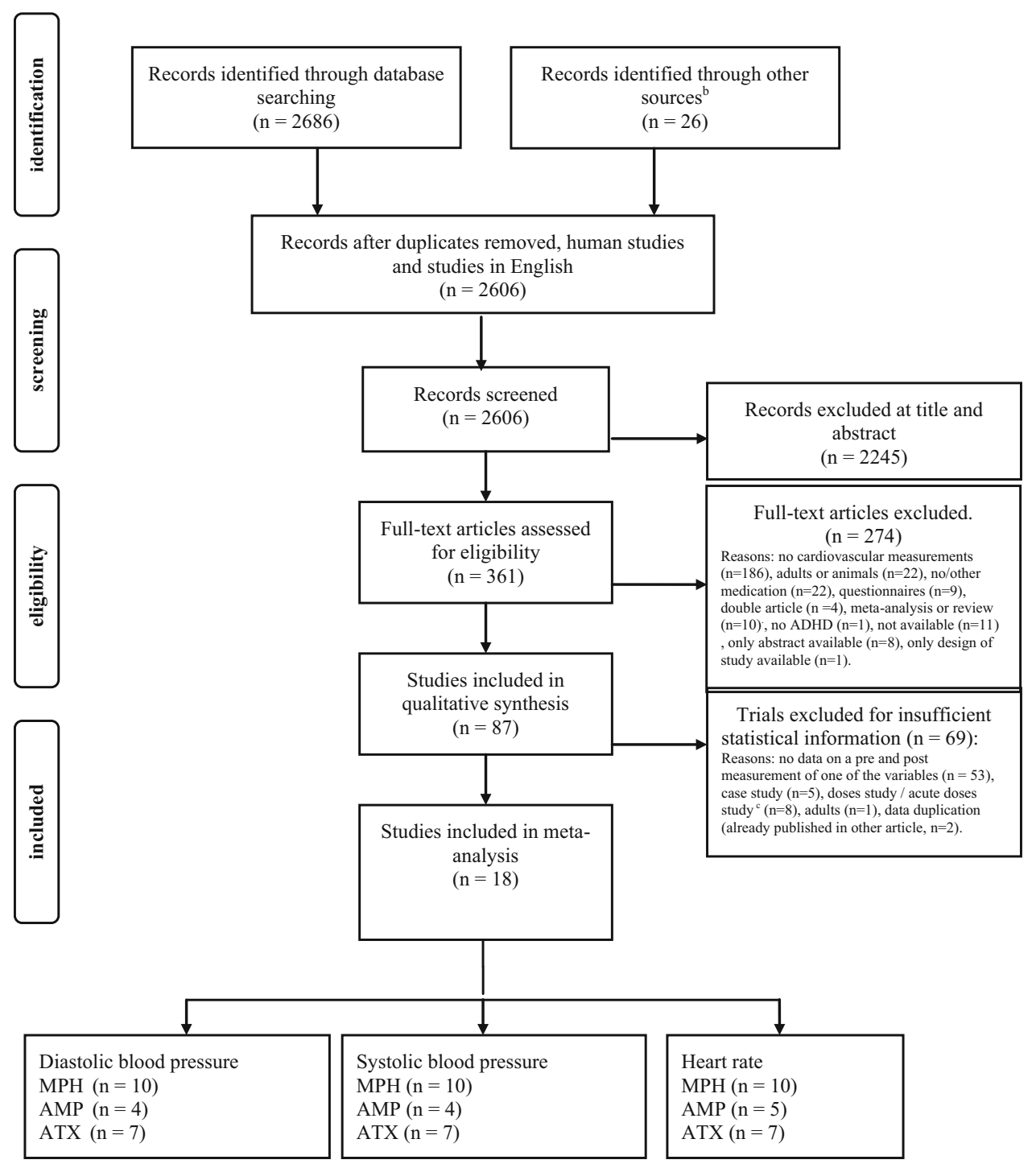




\subsection{Outcome Measures}

The outcome measures were the pre-post treatment change in DBP, SBP and HR. Throughout this paper, when reference is made to SBP, DBP or HR, the data presented will be mean results, unless otherwise stated. Where two or more papers had been written from the same dataset, the outcomes of the longest follow-up were recorded in order to explore measurements of cardiovascular parameters over the longest follow-up interval. Where studies $(n=3)$ included cardiovascular parameters from both a medication and a comparison arm (e.g., placebo or control group), only the parameters for the medication treatment group were included in the analysis. HR is reported from either an ECG-measured HR or a manually measured pulse rate. Where a study reported both types of measure at the same time point, we used the ECG measurement as it was deemed likely to be more accurate.

We also reviewed reporting of other 'cardiovascular effects' at the level of the individual. These were not strictly defined but incorporated any adverse reaction or symptom that was reported including clinical hypertension and heart rate $>90$ th percentile, tachycardia, bradycardia, documented arrhythmia and changes in ECG intervals, morphology or repolarisation. It was important that studies had performed baseline cardiovascular measurements prior to starting medication, in order to give an accurate assessment of change and to ensure that any pre-existing cardiovascular disorders/illnesses/complaints were identified before the study started.

\subsection{Study Selection}

Titles and abstracts of articles were checked by at least two of the authors (LH, MJB, JKB) if they met the inclusion criteria. Assessment of articles for final inclusion was based on full-text revision.

\subsection{Data Extraction}

Sample information and the cardiovascular data of the included trials were entered into Review Manager version 5.3 (http://ims.cochrane.org/revman). This provided a systematic record of study features. Effect sizes from Review Manager and possible moderators of the cardiovascular parameters (type of medication [e.g. MPH, AMP, ATX], doses, duration of treatment, sample size, age, male percentage, type of ADHD, comorbidity, dropout rate and publication year) were extracted into SPSS version 20.

\subsection{Statistical Analyses}

The pre-post within-group design was used to analyse medication effects on DBP, SBP and HR. Individual effect sizes (ESs, expressed as the standard mean difference [SMD]) were calculated in Review Manager version 5.3. The ES is the difference between the pre-treatment and the post-treatment divided by the pooled pre-treatment and post-treatment standard deviation. The ES can be considered to be a small (SMD $>0.2$ ), medium ( $\mathrm{SDM}>0.5$ ) or large (SMD >0.8) [28]. Given the heterogeneity of sample characteristics and implementation of treatments in the included studies, we chose, a priori, to use a random effects model, as recommended by Field and Gillett [29]. Heterogeneity was calculated using $\chi^{2}$ and $I^{2}$ tests with the $I^{2}$ statistic used as an estimate of between-trial heterogeneity in SMD and the $\chi^{2}$ test as an index of whether medication type (e.g. MPH, AMP, ATX), dose, duration of treatment, sample size, age, gender, type of ADHD, comorbidity, dropout rate and publication year had an effect on the SMD. Dummy variables by median split were calculated for age, gender (male percentage), type of ADHD, comorbidity (yes/no), dosage, duration of treatment and publication year. Median split was chosen instead of the mean because of the effect of outliers. When in the results section the word 'significant' is used, we are referring to statistically significant effects and not clinically significant effects, unless otherwise stated.

\section{Results}

Table 1 summarises the participants and study characteristics of all the included studies in this meta-analysis. Eighteen trials [30-47] (from 2712 records: database searching $[n=2686]$ and other sources $[n=26])$ met the inclusion criteria. These reported data from 5837 participants (mean age 10.5 years, range 3.5-18 years; $80.7 \%$ males). Six studies reported effects for MPH alone [30-35], four for AMP alone [36-39] and three for ATX alone [40-42]. Four reported data on both MPH and ATX [43-46] and one on both AMP and ATX [47].

Fourteen studies employed an open-label design [30, 33-37, 39-44, 46, 48], while the remainder had a double-blind, randomised control design [31, 38, 45, 47]. In total, five studies included a control group in their design [31, 35, 38, 45, 47]. Despite including a control group, two studies [31, 45] did not report cardiovascular parameters for the control group. Another study [38] reported only data for a safety population including medication and control group data.

Fourteen out of 18 trials [30, 33, 36-47] specified the distribution of ADHD subtypes and included participants of the different subtypes: inattentive $(n=14)$, hyperactive-impulsive $(n=13)$ and combined type $(n=14)$. Comorbidity was reported in 12 studies [30, 31, 35, $37,41-48]$, with the most commonly reported conditions 
Table 1 Summary of characteristics of studies included in meta-analyses with cardiovascular parameters

\begin{tabular}{|c|c|c|c|c|c|c|c|c|}
\hline Study & $\begin{array}{l}\text { Type of } \\
\text { medication }\end{array}$ & Study design & $\begin{array}{l}\text { Sample } \\
\text { size, } \\
N \text { (drop- } \\
\text { out } \%)\end{array}$ & $\begin{array}{l}\text { Mean age, } \\
\text { y (range) }\end{array}$ & $\begin{array}{l}\text { Male, } \\
\%\end{array}$ & $\begin{array}{l}\text { Type of } \\
\text { comparison }\end{array}$ & $\begin{array}{l}\text { Doses, mean } \\
{[\mathrm{SD}] \text { or range }} \\
\text { per day }\end{array}$ & $\begin{array}{l}\text { Length of } \\
\text { study/ } \\
\text { observation, } \\
\text { weeks }\end{array}$ \\
\hline \multicolumn{9}{|l|}{ MPH } \\
\hline $\begin{array}{l}\text { Buitelaar } \\
\text { et al. [31] }\end{array}$ & MPH & $\begin{array}{l}\text { Prospective, double- } \\
\text { blind placebo- } \\
\text { controlled study }\end{array}$ & 46 (NR) & $9.3(7-13)$ & 88 & $\begin{array}{l}\text { Pindolol and } \\
\text { placebo }\end{array}$ & $20 \mathrm{mg}$ & 4 \\
\hline $\begin{array}{l}\text { Hammerness } \\
\text { et al. [48] }\end{array}$ & $\begin{array}{r}\text { OROS- } \\
\text { MPH }\end{array}$ & Open-label study & $114(50)$ & $\begin{array}{l}14.1 \\
\quad(12-18)\end{array}$ & 73 & NR & $\begin{array}{l}0.5-1.75 \mathrm{mg} / \\
\mathrm{kg}\end{array}$ & 24 \\
\hline $\begin{array}{l}\text { Kim et al. } \\
{[30]}\end{array}$ & $\begin{array}{r}\text { OROS- } \\
\text { MPH }\end{array}$ & $\begin{array}{l}\text { Prospective, open- } \\
\text { label, flexible-dose }\end{array}$ & $\begin{array}{l}24 \\
(11.1)\end{array}$ & $8.2(6-12)$ & 92 & NR & $18-45 \mathrm{mg}$ & 24 \\
\hline $\begin{array}{l}\text { Lee et al. } \\
\text { [33] }\end{array}$ & $\begin{array}{r}\text { OROS- } \\
\text { MPH }\end{array}$ & Open-label study & $\begin{array}{l}47 \\
(14.5)\end{array}$ & $\begin{array}{l}14.3 \\
(12-18)\end{array}$ & 78 & NR & $18-72 \mathrm{mg}$ & 12 \\
\hline $\begin{array}{l}\text { Wilens et al. } \\
\text { [34] }\end{array}$ & $\begin{array}{r}\text { OROS- } \\
\text { MPH }\end{array}$ & Open-label study & $432(29)$ & NR (6-13) & NR & NR & $18-54 \mathrm{mg}$ & 48 \\
\hline Zeiner [35] & MPH & Open-label study & $23(13)$ & $9.3(7-12)$ & 100 & No medication & $0.55 \mathrm{mg} / \mathrm{kg}$ & 84 \\
\hline \multicolumn{9}{|l|}{ AMP } \\
\hline $\begin{array}{l}\text { Coghill et al. } \\
\text { [36] }\end{array}$ & LDX & Open-label trial & $\begin{array}{l}276 \\
(39.9)\end{array}$ & $\begin{array}{l}10.9 \\
(6-17)\end{array}$ & 76.8 & Placebo & $30-70 \mathrm{mg}$ & 52 \\
\hline $\begin{array}{l}\text { Donner et al. } \\
\text { [37] }\end{array}$ & MAS XR & $\begin{array}{l}\text { Prospective, open- } \\
\text { label, no } \\
\text { comparative, } \\
\text { community-based } \\
\text { study }\end{array}$ & $\begin{array}{l}2968 \\
(1.2)\end{array}$ & $9.5(6-13)$ & 76.1 & Doses & $10-40 \mathrm{mg}$ & 14 \\
\hline $\begin{array}{l}\text { Findling } \\
\text { et al. [38] }\end{array}$ & LDX & $\begin{array}{l}\text { Open-label, } \\
\text { multicentre, } \\
\text { randomised, double- } \\
\text { blind, placebo- } \\
\text { controlled study }\end{array}$ & $314(42)$ & $\begin{array}{l}14.5 \\
\quad(13-17)\end{array}$ & 70.6 & Placebo (no data) & $30-70 \mathrm{mg}$ & 52 \\
\hline $\begin{array}{l}\text { Wilens et al. } \\
{[39]^{\mathrm{b}}}\end{array}$ & MAS XR & Open-label study & $\begin{array}{l}138 \\
(\mathrm{NR})\end{array}$ & 14.4 & 71.0 & NR & $10-40 \mathrm{mg}$ & 16 \\
\hline \multicolumn{9}{|l|}{ ATX } \\
\hline $\begin{array}{l}\text { Fuentes et al. } \\
\text { [40] }\end{array}$ & ATX & $\begin{array}{l}\text { Randomised, } \\
\text { controlled, open-label } \\
\text { study }\end{array}$ & $\begin{array}{l}199 \\
(21.1)\end{array}$ & $9.2(6-16)$ & 79.4 & $\begin{array}{l}\text { Any other } \\
\text { pharmacological } \\
\text { ADHD treatment }\end{array}$ & $0.5-1.8 \mathrm{mg} / \mathrm{kg}$ & 48 \\
\hline $\begin{array}{l}\text { Ghuman } \\
\text { et al. [41] }\end{array}$ & ATX & Open-label pilot study & $12(0)$ & $\begin{array}{l}5.0 \\
\quad(3.5-5.8)\end{array}$ & 75 & NR & $\begin{array}{l}\text { Up titration } \\
18-40 \mathrm{mg}\end{array}$ & 6 \\
\hline $\begin{array}{l}\text { Hammerness } \\
\text { et al. [42] }\end{array}$ & ATX & Two-phase open study & $\begin{array}{l}72 \\
(16.7)\end{array}$ & $9.3(6-17)$ & 76 & $\begin{array}{l}\text { ATX (vs ATX and } \\
\text { OROS-MPH) }\end{array}$ & $\begin{array}{l}\text { ATX } \\
0.5-1.4 \mathrm{mg} / \\
\text { kg } \\
\text { OROS-MPH } \\
18 / 54 \mathrm{mg}\end{array}$ & 4 \\
\hline \multicolumn{9}{|l|}{$>1$ medication } \\
\hline $\begin{array}{l}\text { Arcieri et al. } \\
\text { [43] }\end{array}$ & $\begin{array}{l}\text { MPH } \\
\text { ATX }\end{array}$ & $\begin{array}{l}\text { Open-label, } \\
\text { prospective, } \\
\text { observational study }\end{array}$ & $\begin{array}{l}351 \\
(82.6) \\
350 \\
(89.1)\end{array}$ & $\begin{array}{l}10.4 \\
(6-18) \\
10.8 \\
(6-18)\end{array}$ & $\begin{array}{l}87 \\
90\end{array}$ & $\begin{array}{l}\text { MPH and } \mathrm{BT}^{\mathrm{a}} \\
\text { ATX and BT }\end{array}$ & $\begin{array}{l}\text { MPH } 18.4 \\
(10.4) \\
\text { ATX } 38.6 \\
(20.5)\end{array}$ & 96 \\
\hline \multirow[t]{2}{*}{$\begin{array}{l}\text { Dittmann } \\
\text { et al. [47] }\end{array}$} & \multirow[t]{2}{*}{$\begin{array}{l}\text { LDX } \\
\text { ATX }\end{array}$} & \multirow{2}{*}{$\begin{array}{l}\text { Head-to-head, } \\
\text { randomised, double- } \\
\text { blind, active- } \\
\text { controlled study }\end{array}$} & $\begin{array}{l}128 \\
(24.8)\end{array}$ & $\begin{array}{l}10.9 \\
(3.01)\end{array}$ & 75.2 & LDX & $\begin{array}{l}\text { LDX } \\
\quad 30-70 \mathrm{mg}\end{array}$ & 9 \\
\hline & & & $\begin{array}{l}134 \\
(24.6)\end{array}$ & $\begin{array}{l}10.4 \\
(2.84)\end{array}$ & 76.9 & ATX & $\begin{array}{r}\text { ATX < } 70 \mathrm{~kg}: \\
0.5-1.4 \mathrm{mg} / \\
\mathrm{kg} ;>70 \mathrm{~kg}: \\
40-100 \mathrm{mg}\end{array}$ & \\
\hline
\end{tabular}


Table 1 continued

\begin{tabular}{|c|c|c|c|c|c|c|c|c|}
\hline Study & $\begin{array}{l}\text { Type of } \\
\text { medication }\end{array}$ & Study design & $\begin{array}{l}\text { Sample } \\
\text { size, } \\
N \text { (drop- } \\
\text { out } \% \text { ) }\end{array}$ & $\begin{array}{l}\text { Mean age, } \\
\text { y (range) }\end{array}$ & $\begin{array}{l}\text { Male, } \\
\%\end{array}$ & $\begin{array}{l}\text { Type of } \\
\text { comparison }\end{array}$ & $\begin{array}{l}\text { Doses, mean } \\
{[\mathrm{SD}] \text { or range }} \\
\text { per day }\end{array}$ & $\begin{array}{l}\text { Length of } \\
\text { study/ } \\
\text { observation, } \\
\text { weeks }\end{array}$ \\
\hline \multirow[t]{2}{*}{$\begin{array}{l}\text { Kratochvil } \\
\text { et al. [44] }\end{array}$} & \multirow[t]{2}{*}{$\begin{array}{l}\text { MPH } \\
\text { ATX }\end{array}$} & \multirow{2}{*}{$\begin{array}{l}\text { A prospective, } \\
\text { randomised, open- } \\
\text { label }\end{array}$} & $\begin{array}{l}40 \\
(37.5)\end{array}$ & $\begin{array}{l}10.4 \\
(7-15)\end{array}$ & 100 & MPH & $5-60 \mathrm{mg}$ & 10 \\
\hline & & & $\begin{array}{l}180 \\
(35.9)\end{array}$ & $\begin{array}{l}10.4 \\
(7-15)\end{array}$ & 90.8 & ATX & $0.2-1.0 \mathrm{mg} / \mathrm{kg}$ & 10 \\
\hline \multirow[t]{2}{*}{$\begin{array}{l}\text { Sangal et al. } \\
\text { [45] }\end{array}$} & \multirow[t]{2}{*}{$\begin{array}{l}\text { MPH } \\
\text { ATX }\end{array}$} & \multirow[t]{2}{*}{$\begin{array}{l}\text { Randomised, double- } \\
\text { blind crossover }\end{array}$} & $83(5.3)$ & $\begin{array}{l}10.1 \\
(6-14)\end{array}$ & 75 & $\mathrm{MPH}$ & $\begin{array}{l}0.45-1.8 \mathrm{mg} / \\
\mathrm{kg}\end{array}$ & 7 \\
\hline & & & 81 (NR) & NR & NR & ATX & NR & 7 \\
\hline \multirow[t]{2}{*}{$\begin{array}{l}\text { Yildiz et al. } \\
{[46]^{\mathrm{b}}}\end{array}$} & $\begin{array}{r}\text { OROS- } \\
\text { MPH }\end{array}$ & Open-label study & $\begin{array}{l}11 \\
(13.3)\end{array}$ & $9.0(8-13)$ & 82 & OROS-MPH & $18-54 \mathrm{mg}$ & 12 \\
\hline & ATX & & $\begin{array}{l}14 \\
(17.6)\end{array}$ & $\begin{array}{l}9.78 \\
\quad(8-12)\end{array}$ & 93 & ATX & $18-60 \mathrm{mg}$ & 12 \\
\hline
\end{tabular}

AMP amphetamines, $A T X$ atomoxetine, $L D X$ lisdexamfetamine dimesylate, $M A S X R$ amphetamine salts extended release, $M P H$ methylphenidate, $N R$ not reported, OROS-MPH osmotic release oral system-MPH (Concerta ${ }^{\circledR}$ ), SD standard deviation

${ }^{a}$ Behaviour treatment (BT) involves child/parent/family training, psychodynamic therapy or counselling

b Study with only heart rate data

being oppositional defiant disorder ( $n=9$ studies), conduct disorder $(n=9)$, anxiety disorder $(n=7)$, depression $(n=5)$ or other disorders $(n=5)$.

Fourteen studies [30, 31, 33-35, 37-39, 42, 45-48] had performed cardiovascular measurements during screening and excluded children with cardiovascular disorders/illnesses/complaints beforehand. Four studies reported no information about the screening process [36, 40, 41, 44].

Trial duration ranged from 4 to 96 weeks (mean 28.7 weeks): $4-12$ weeks $(n=8), 13-51$ weeks $(n=6)$ and $>52$ weeks $(n=4)$. The average duration for MPH studies was 28.1 weeks (range 4-96), for AMP studies 29.2 weeks (range 9-52) and for ATX studies 24 weeks (range 4-96). Seven studies [34, 37-40, 43, 48] reported multiple follow-up outcome measurements. The outcome of the latest follow-up was recorded. The average drop-out rate was $1149 / 5836$ (19.9\%; 18 studies). Two studies were published before 2000, nine between 2000 and 2010, and seven after 2010.

\subsection{Diastolic Blood Pressure}

AMP (SMD 0.16, 95\% confidence interval [CI] 0.03-0.29, $p=0.02$; four studies) and ATX (SMD 0.22, 95\% CI $0.10-0.34, p<0.01$; seven studies) were associated with small but statistically significant pre-post differences. The effect for MPH was not significant (SMD 0.16, 95\% CI 0.04 to $0.36, p=0.11$; ten studies). When effects were pooled for all medications together, there was a small but statistically significant increase in DBP associated with ADHD medication (SMD 0.18, 95\% CI 0.09-0.26, $p<0.01 ; 17$ studies) and no significant difference between the three medications when comparing two drugs per comparison.

\subsection{Systolic Blood Pressure}

MPH (SMD 0.25, 95\% CI 0.08-0.42, $p<0.01$; ten trials), AMP (SMD 0.09, 95\% CI 0.03-0.15, $p<0.01$; four studies) and ATX (SMD 0.16, 95\% CI 0.04-0.27, $p=0.01$; seven studies) each had small but statistically significant pre-post effects on SBP. Across all medications together there was a small and significant pre-post effect (SMD $0.18,95 \%$ CI $0.10-0.27, p<0.01 ; 17$ studies) with no differences between the three medications when comparing two drugs per comparison.

\subsection{Heart Rate}

For HR there were small but significant effects for AMP (SMD 0.37, 95\% CI 0.13-0.60, $p<0.01$; five studies) and ATX (SMD 0.43, 95\% CI 0.26-0.60, $p<0.01$; seven studies) but the effects for MPH were not statistically significant (SMD 0.20, 95\% CI -0.02 to $0.41, p=0.07$; 10 studies). Pooled pre-post effects across all three medications were small but statistically significant (SMD 0.32, 95\% CI $0.20-0.43, p<0.01 ; 17$ studies) on HR. Figure 2 shows the forest plots for all three medications and the cardiovascular parameters.

\subsection{Multiple Measurements Over Time}

For MPH, three studies [34, 43, 48] reported data of multiple measurements (number of measurements 3-5; 
DBP

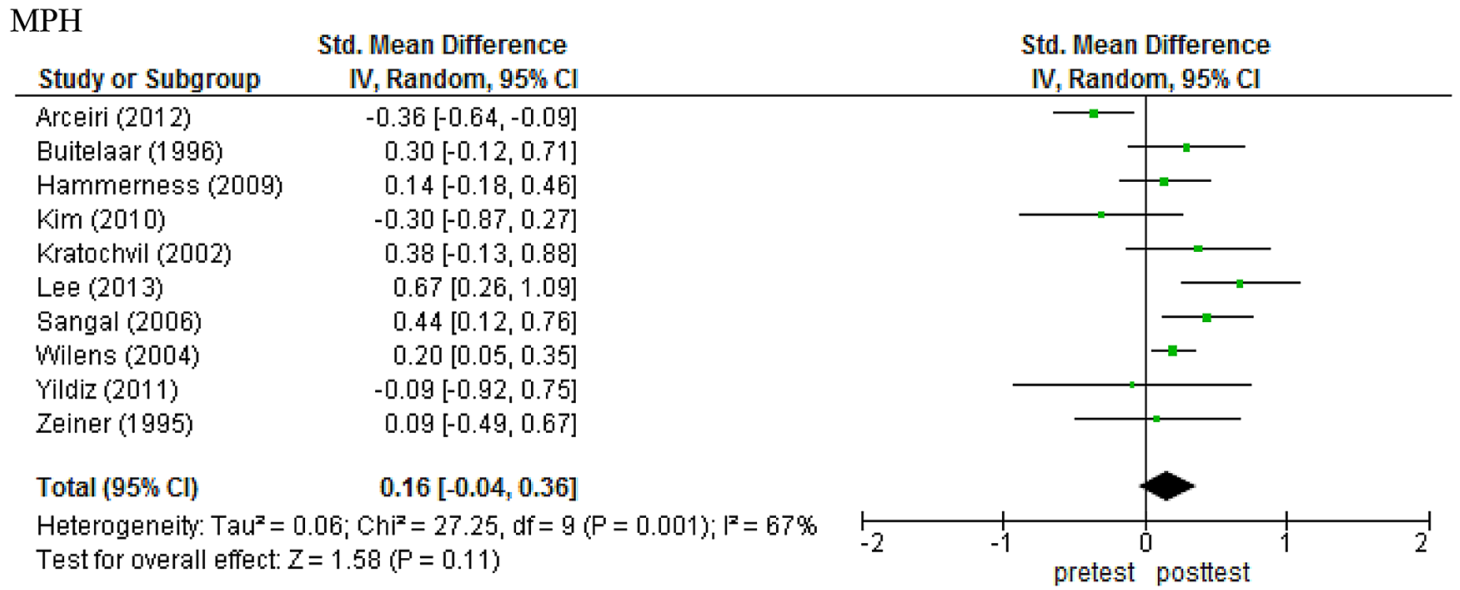

AMP

\begin{tabular}{|c|c|c|c|c|}
\hline Study or Subgroup & $\begin{array}{l}\text { Mean Difference } \\
\mathrm{V} \text {, Random, } 95 \% \mathrm{Cl}\end{array}$ & \multicolumn{3}{|c|}{$\begin{array}{l}\text { Std. Mean Difference } \\
\text { IV, Random, } 95 \% \mathrm{Cl}\end{array}$} \\
\hline Coghill (2014) & $0.24[0.07,0.40]$ & & \multicolumn{2}{|c|}{$\mid--$} \\
\hline Dittmann (2013) & $0.01[-0.23,0.26]$ & & \multirow{2}{*}{\multicolumn{2}{|c|}{ E }} \\
\hline Donner (2007) & $0.08[0.03,0.13]$ & & & \\
\hline Findling (2013) & $0.32[0.14,0.51]$ & & \multicolumn{2}{|l|}{-1} \\
\hline Total $(95 \% \mathrm{Cl})$ & $0.16[0.03,0.29]$ & & & \\
\hline $\begin{array}{l}\text { Heterogeneity: Tau } \\
\text { Test for overall effe }\end{array}$ & $\begin{array}{l}\mathrm{Chi}^{2}=8.91, \mathrm{df}=3(\mathrm{P}=0.03) ; I^{2}=66 \% \\
.43(\mathrm{P}=0.02)\end{array}$ & \multicolumn{3}{|c|}{ pretest posttest } \\
\hline
\end{tabular}

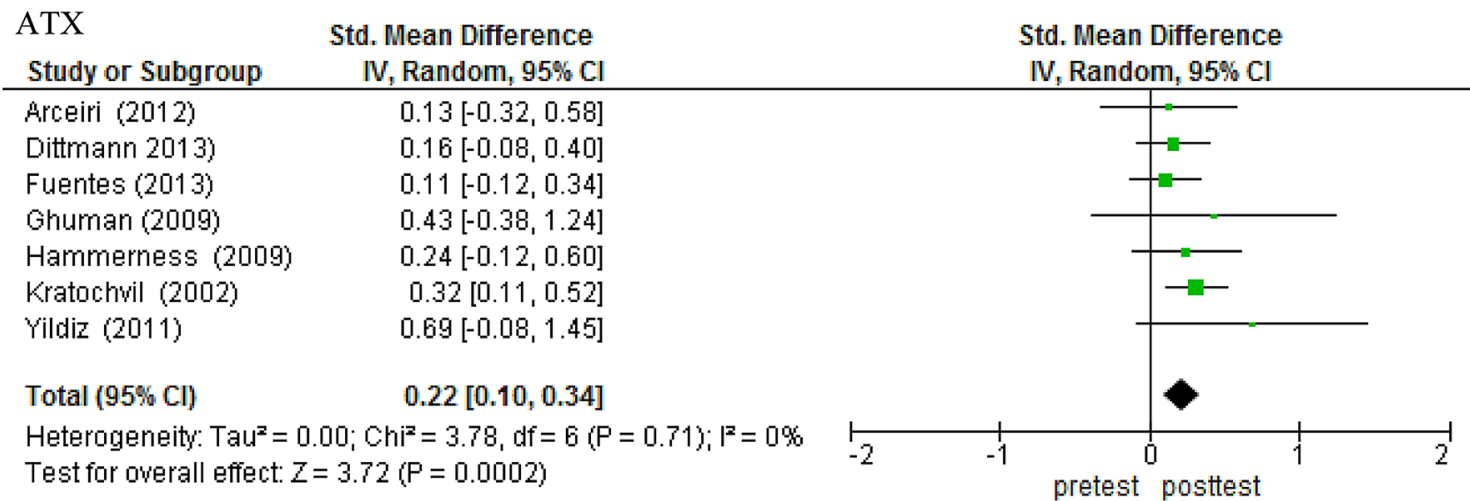

\footnotetext{
All medications
Total $(95 \% \mathrm{Cl})$
$0.18[0.09,0.26]$
Heterogeneity: Tau $=0.02 ; \mathrm{Chi}^{2}=43.64, \mathrm{df}=20(\mathrm{P}=0.002) ; \mathrm{I}^{2}=54 \%$
Test for owerall effect: $Z=4.13$ ( $\mathrm{P} \leq 0.0001)$

Fig. 2 Forest plots with pre-post standardised mean differences SMDs (ES) and homogeneity statistics for meta-analyses of DBP, SBP and HR. The forest plots represent each study in the metaanalysis, plotted according to the SMD. The SMD is the difference between the pre-test and the post-test divided by the pooled pre-test and post-test standard deviation. The green box on each line shows the SMD for each study. The size of the box stands for the size of the sample size. The black diamond at the bottom of the graph shows the average SMD of all studies of all medications. If a green box or the black diamond stands on the left side of the middle line, this represents a higher $\mathrm{DBP}, \mathrm{SBP}$ or $\mathrm{HR}$ on the pre-test in comparison with the post-test, so a decrease. A box/diamond on the right side of the middle line represents a higher $\mathrm{DBP}, \mathrm{SBP}$ or $\mathrm{HR}$ on the post-test in comparison with the pre-test, so an increase. If the green box or the black diamond crosses the middle line, then this study reported no significant effect. For more explanation about forest plots, see [58]. $A M P$ amphetamines, $A T X$ atomoxetine, $C I$ confidence interval, $D B P$ diastolic blood pressure, $d f$ degrees of freedom, $E S$ effect size, $H R$ heart rate, $M P H$ methylphenidate, $S B P$ systolic blood pressure, $S M D$ standard mean difference 
SBP

$\mathrm{MPH}$

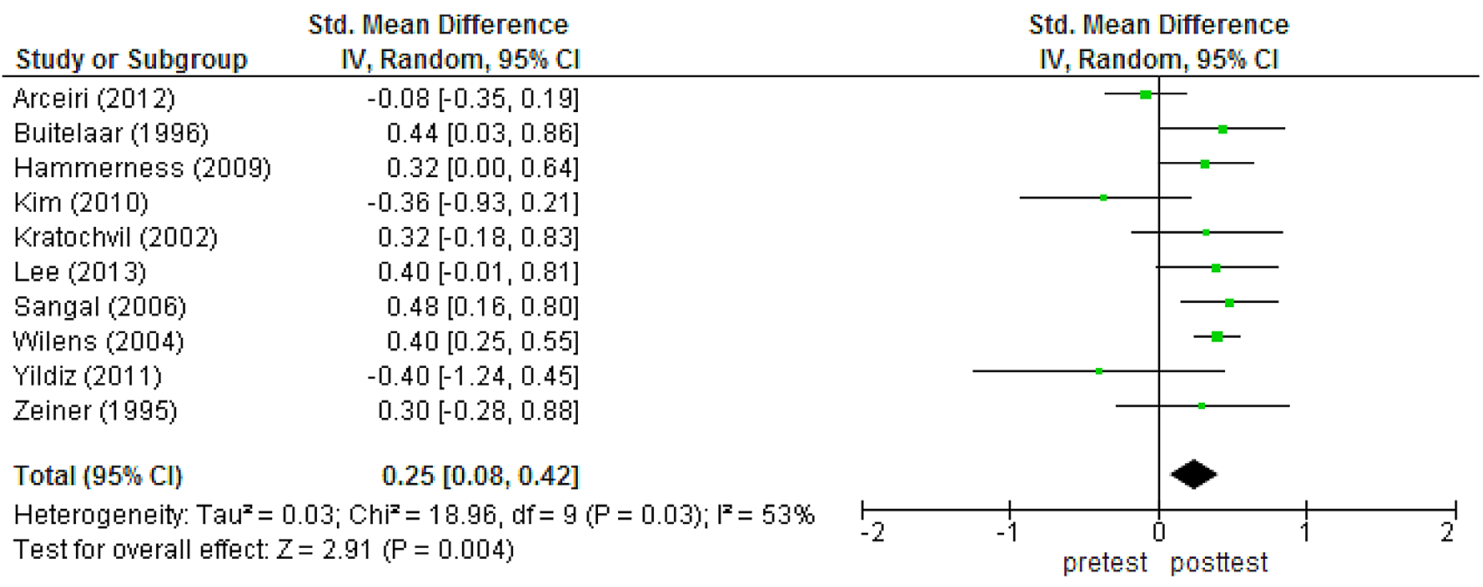

AMP

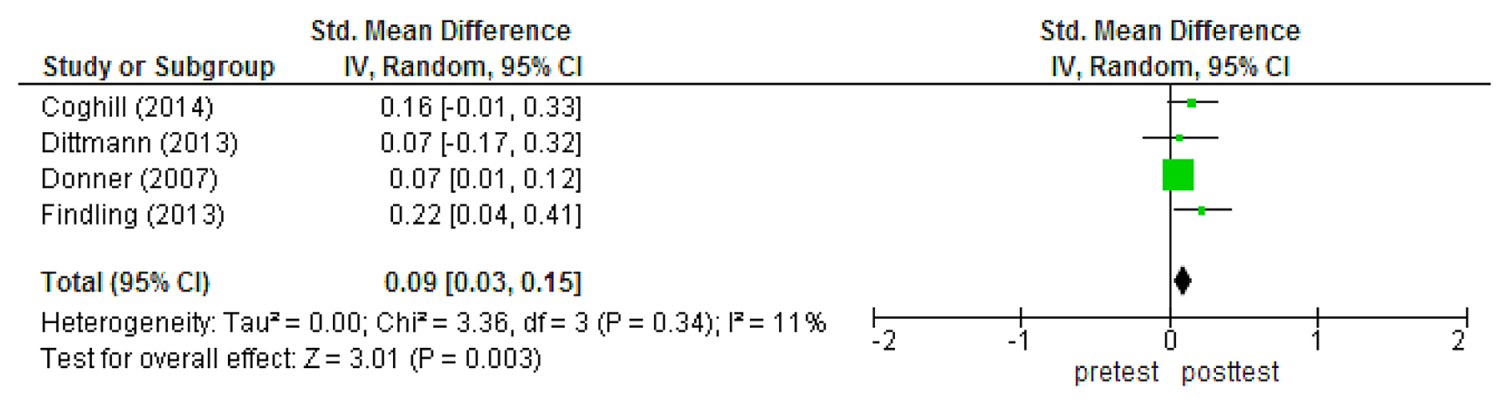

ATX

Std. Mean Difference

Std. Mean Difference

Study or Subgroup

IV, Random, $95 \% \mathrm{Cl}$

IV, Random, $95 \% \mathrm{Cl}$

$\begin{array}{lr}\text { Arceiri (2012) } & 0.21[-0.24,0.66] \\ \text { Dittmann 2013) } & 0.07[-0.17,0.31] \\ \text { Fuentes (2013) } & 0.08[-0.15,0.32] \\ \text { Ghuman (2009) } & -0.03[-0.83,0.77] \\ \text { Hammeness (2009) } & 0.15[-0.21,0.51] \\ \text { Kratochil }(2002) & 0.27[0.06,0.48] \\ \text { Yildiz (2011) } & 0.37[-0.38,1.11] \\ & \\ \text { Total (95\% Cl) } & 0.16[0.04,0.27] \\ \text { Heterogeneity: Tau }{ }^{2}=0.00 ; \mathrm{Ch}^{2}=2.64, \mathrm{df}=6(\mathrm{P}=0.85) ; \mathrm{I}^{2}=0 \% \\ \text { Test for overall effect: } Z=2.67(\mathrm{P}=0.008)\end{array}$

$0.07[-0.17,0.31]$

$0.08[-0.15,0.32]$

$0.03[-0.83,0.77$

$.27[0.06,0.48]$

$0.16[0.04,0.27]$

Test for overall effect: $Z=2.67(\mathrm{P}=0.008)$

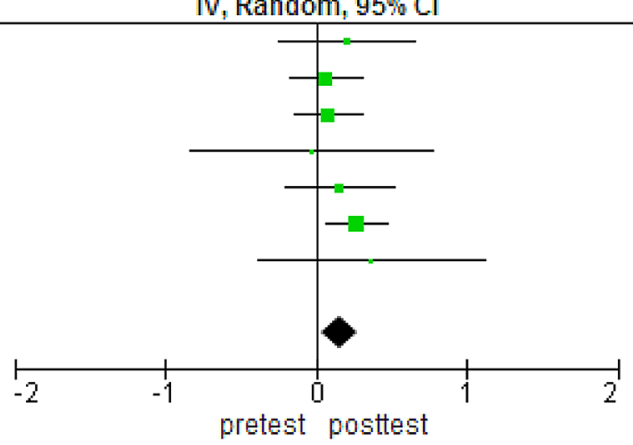

All medications

Total $(95 \% \mathrm{Cl})$

$0.18[0.10,0.27]$

Heterogeneity: Tau ${ }^{2}=0.01 ; \mathrm{Ch}^{2}=40.35, \mathrm{df}=20(\mathrm{P}=0.005) ; \mathrm{I}^{2}=50 \%$

Test for owerall effect: $Z=4.41(\mathrm{P}=0.0001)$

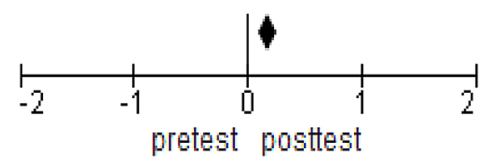

Fig. 2 continued 


\section{HR}

\section{$\mathrm{MPH}$}

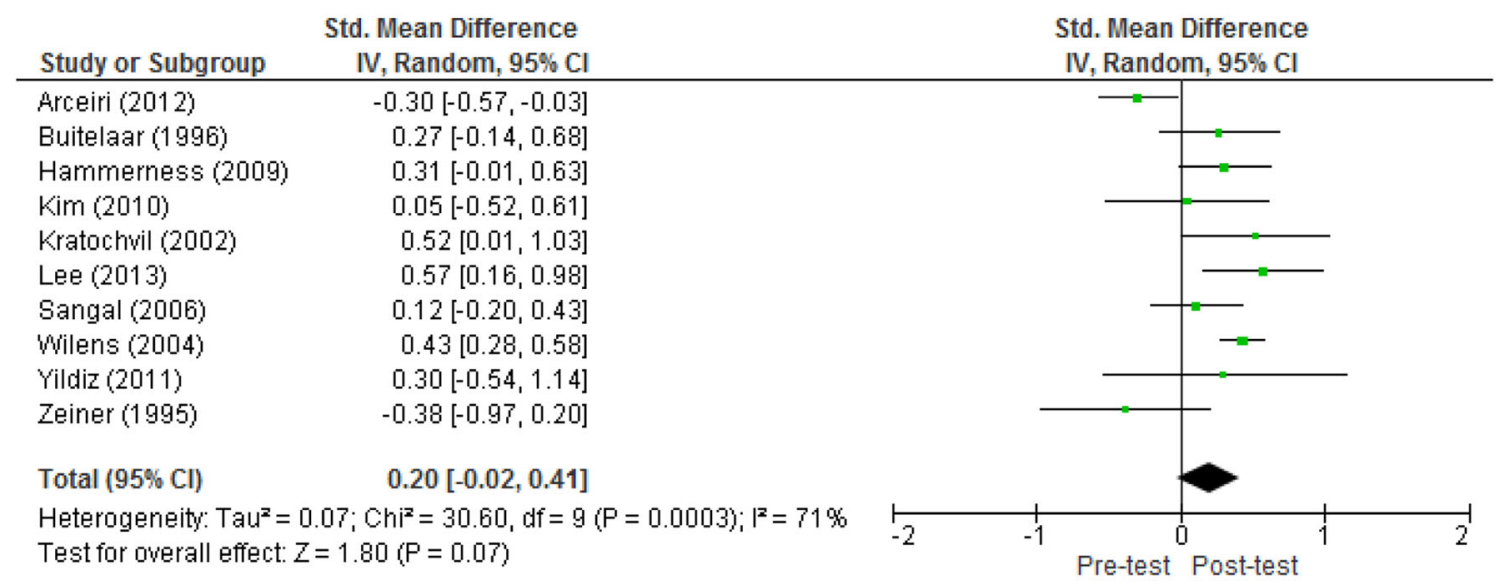

AMP

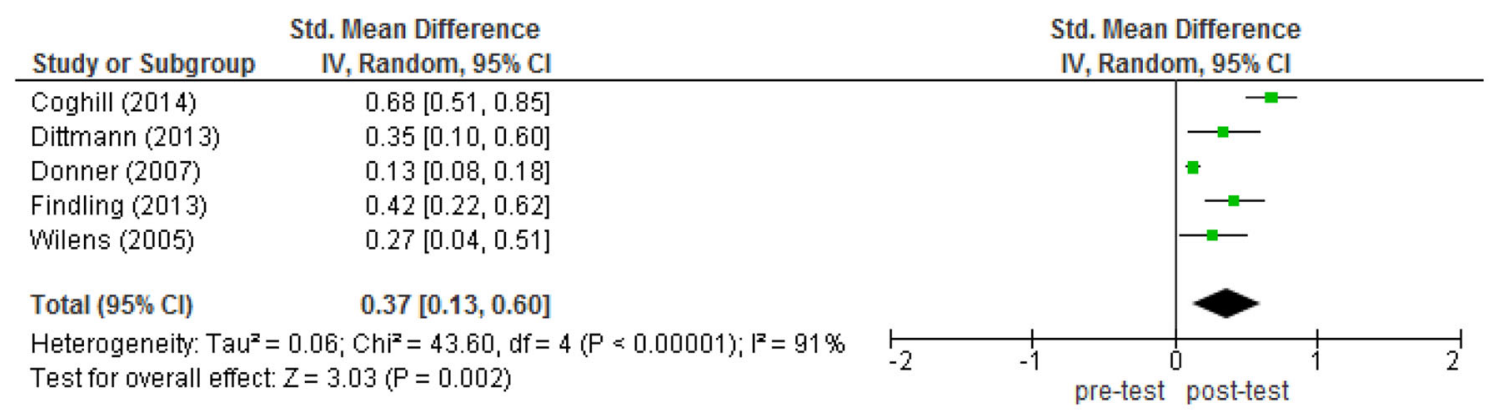

Std. Mean Difference

Std. Mean Difference

IV, Random, $95 \% \mathrm{Cl}$

$0.02[-0.32,0.35]$

$0.37[0.13,0.61]$

$0.59[0.35,0.82]$

$0.45[-0.36,1.26]$

$0.73[0.36,1.11]$

$0.49[0.28,0.70]$

$0.17[-0.57,0.91]$

$0.43[0.26,0.60]$

Total $(95 \% \mathrm{Cl})$

Heterogeneity: Tau $^{2}=0.02 ; \mathrm{Chi}^{2}=10.98, \mathrm{df}=6(\mathrm{P}=0.09) ; \mathrm{I}^{2}=45 \%$

Test for overall effect: $Z=4.98(P=0.00001)$

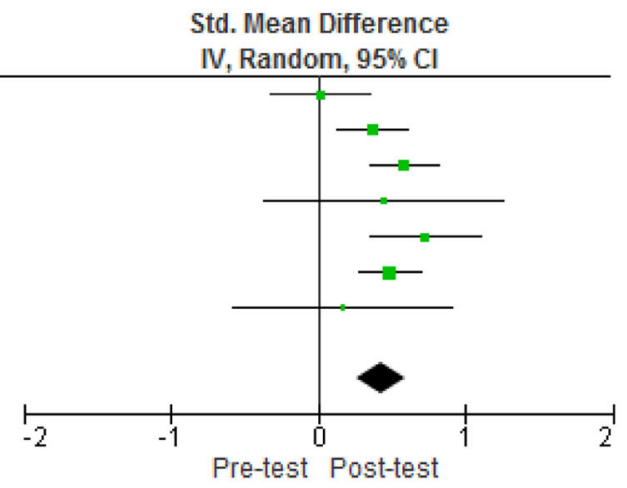

All medications:

Total $(95 \% \mathrm{Cl})$

$0.32[0.20,0.43]$

Heterogeneity: $\mathrm{Tau}^{2}=0.05 ; \mathrm{Chi}^{2}=101.02, \mathrm{df}=21(\mathrm{P}=0.00001) ; \mathrm{I}^{2}=79 \%$

Test for overall effect: $Z=5.25$ ( $P<0.00001)$

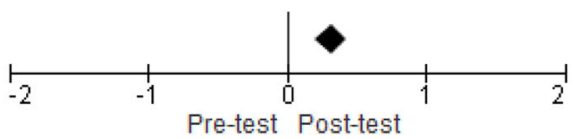

Fig. 2 continued

duration range 24-96 weeks). A peak in DBP (mean $+1.7 \mathrm{mmHg}$ ) and $\mathrm{HR}($ mean $+3.7 \mathrm{bpm}$ ) was reported at the first measurement (average 3.2 months after baseline) compared with baseline and follow-up measurements. Similar results were reported on SBP (mean $+1.6 \mathrm{mmHg})$. 
Two AMP studies reported a trend of increased DBP/ SBP/HR until 14 weeks [37] and 52 weeks of treatment [38]. Once again, the highest peak was at the first measurement after baseline (average 6 weeks; mean changes: $\mathrm{DBP}+1 \mathrm{mmHg}$; SBP +0.75 mmHg; HR +3.35 bpm).

Arcieri et al. [43] and Fuentes et al. [40] studied ATX and reported the highest effect in DBP, SBP and HR at the first measurement (at 6 months after baseline); effects on DBP $(+0.2 \mathrm{mmHg})$ and $\mathrm{HR}(+2.9 \mathrm{bpm})$ were statistically significant, while effect on SBP $(+0.8 \mathrm{mmHg})$ was not significant in Arcieri et al. [43]. Despite the increased levels at the first measurement, Arcieri et al. [43] reported no statistically significant effects on DBP $(+0.13 \mathrm{mmHg})$ and SBP $(+0.36 \mathrm{mmHg})$ at 12 months in comparison with baseline, only HR $(+3.26 \mathrm{bpm})$ was statistically significant. There were no statistically significant effects at 24 months in comparison with the baseline (DBP $+1.11 \mathrm{mmHg}$; SBP +2.13 mmHg; HR +0.21 bpm). Fuentes et al. [40] observed small changes at 6 months for all cardiovascular parameters (DBP +1.3 mmHg; SBP +0.8 mmHg; $\mathrm{HR}+7.9 \mathrm{bpm}$ ) and at 12 months (DBP $+1.6 \mathrm{mmHg}$; $\mathrm{SBP}+1.6 \mathrm{mmHg}$; $\mathrm{HR}$ $+5.6 \mathrm{bpm}$ ) compared with baseline. Only the mean changes for HR were reported as significant.

In summary, the largest change in studies with multiple measurement points was overall reported at the first measurement time point after baseline (average 13.3 weeks; 7 studies).

\subsection{Moderators of the Treatment Effect}

Sensitivity analyses revealed a significant effect of study duration on DBP following AMP treatment $(F(1)=19.55$, $p=0.05$ ). Studies $<18$ weeks reported higher ESs (SMD 0.28) compared with longer duration studies (SMD 0.05). There was a significant effect of publication year on SBP for MPH treatment $(F(1)=5.346, p=0.05)$. Studies published before 2007 reported higher ESs (SMD 0.37) than studies after 2007 (SMD -0.02). There was no effect of the following moderators: type of medication (e.g. MPH, AMP, ATX), doses, sample size, age, gender (male percentage), type of ADHD, comorbidity and dropout rate. Due to the homogeneity among studies on age, distribution of gender, ADHD type (yes/no), comorbidity (yes/no) and doses, it was not possible to explore their status as moderating influences. It was also not possible to explore the possible influences of comorbidity because of the small samples for each medication.

\subsection{Cardiovascular Adverse Effects on Individual Level}

A majority of studies $(15 / 18,83.3 \%)$ reported individuallevel data about other cardiovascular effects (see Table 2).
Cardiovascular effects reported were hypertension and HR above 90th percentile, tachycardia, brachycardia, arrhythmia, palpitations and ECG abnormalities. Overall, 737 cardiovascular adverse effects were reported in these studies for all 5837 participants (12.6\%). Studies about MPH reported 149/1171 (12.7\%) cardiovascular effects, AMP 462/3705 (12.5\%) and ATX 126/961 (13.1\%). There were no significant differences between the medication treatments, $(F(2)=3.006, p=0.007)$. Notably, the vast majority of patients continued their medication treatment following the report of a cardiovascular adverse event; these resolved spontaneously or medication doses were changed or the events were not considered to be clinically relevant. In case of other cardiovascular effects, 15/737 (2.0\%) discontinued the treatment of MPH $(5 / 149,3.4 \%)$, AMP $(9 / 462,2.0 \%)$ or ATX $(1 / 126,0.8 \%)$. Two participants discontinued their treatment at 6 months and one at 6 weeks; the moment of discontinuation was not reported for the remaining 12 participants (see Table 2, last column, for the reasons why these 15 participants discontinued their medication).

\section{Discussion}

This report presents the first meta-analysis on the effects of treatment with MPH, AMP and ATX on DBP, SBP and HR in children and adolescents with ADHD (see [17] for a review of adult data). Relatively small but statistically significant pre-post increases of BP and HR were associated with medication treatment in children and adolescents with ADHD. All three medications were associated with numerically small but statistically significant elevated SBP. AMP and ATX, but not MPH, were associated with small but statistically significant elevated DBP and HR. There were no statistically significant differences between the medications in their amount of change on cardiovascular outcomes.

Pooled results from the few studies that reported multiple measurements over time showed that the largest changes for DBP, SBP and HR were observed between baseline and the first recorded time point after baseline, indicating a stronger effect in the short term with a tendency towards normalisation thereafter. This should be interpreted cautiously, however. In one study, only $1 / 10$ (38/316 at 6 months) of the subjects were assessed at 24-month follow-up, with no difference compared with baseline for all three measures (SBP, SBP and HR) [43]. Since this was an observational study, it is not clear whether the absence of long-term effects is related to the development of tolerance for these effects or to the fact that patients with earlier significant cardiovascular changes withdrew from the respective medication treatment. 
Table 2 Summary of all cardiovascular effects reported in studies included in this meta-analysis

\begin{tabular}{lllll}
\hline Study & $\begin{array}{l}\text { Hypertension and heart rate } \\
>90 \text { th percentile }\end{array}$ & Tachycardia & Brachycardia & $\begin{array}{l}\text { Other } \\
\text { cardiovascular } \\
\text { effects }\end{array}$
\end{tabular}

\section{$\mathrm{MPH}$}

Arcieri et al. NR [43]

Buitelaar

et al. [31]

Hammerness

et al. [32]

$$
\text { NR }
$$

$6 \%(n=7$; probably because of 0 high BMI)

Kim et al. $\quad 0$

[30]

Kratochvil

et al. [44]

Lee et al. NR

[33]

Sangal et al. NR

[55]

Wilens et al. $1 \mathrm{SBP}>130 \mathrm{mmHg}$ [34]

\section{Yildiz et al. NR}

[46]

Zeiner [35] NR

AMP

Coghill et al. NR

[56]

Dittmann

$11 / 94(11.7 \%)$ DBP $>80 \mathrm{mmHg} \quad \mathrm{NR}$

et al. [47]

$12 / 94(12.8 \%)>$ SBP

$120 \mathrm{mmHg}$

4/127 (3.1\%) HR $<50$ bpm

19/127 (15.0\%) HR >100 bpm

Donner et al. $2.5 \%$ SBP or DBP values that

$$
\begin{aligned}
& \text { were }>95 \text { th percentile } \\
& 3.6 \% \text { had an } \mathrm{HR} \\
& \text { increase }>25-110 \mathrm{bpm} \\
& 6 \mathrm{DBP}>90 \mathrm{mmHg} \\
& 22 \mathrm{SBP}>130 \mathrm{mmHg} \\
& 30 \mathrm{HR}>120 \mathrm{bpm} \\
& 0 \mathrm{HR}<50 \mathrm{bpm}
\end{aligned}
$$

$\begin{array}{cc}6 / 351 & 10 / 351 \\ (1.7 \%) & (2.8 \%)\end{array}$

$(2.8 \%)$

NR

$$
\begin{gathered}
1 / 351(0.3 \%) \\
\text { arrhythmia }
\end{gathered}
$$

NR

NR

0

0

0

$2 / 40(5 \%)$

0

0

0

NR

NR

NR

NR

$0 \%$

$0 \%$

0

$0 \%$

NR

NR

0

NR

NR

NR

NR

NR

NR

NR

NR

NR

$2(0.1 \%)$

NR

7/2968 (0.2\%)

including

hypertension, palpitations, and

tachycardia

(no numbers

of events

separately)
$4.7 \%$ (6 mo), $10 \%$

(12 mo), $10.4 \%$

(24 mo)

5/351 (1.7\%)

lengthened QTc

No ECG

$0 \%$

$0 \%$

$0 \%$

No ECG

$0 \%$

$0 \%$

$0 \%$

No ECG

No ECG

$8 / 83$ (9.6\%)

$\mathrm{HR}>100 \mathrm{bpm}$

QTcF interval change from screening $>30 \mathrm{~ms}$ or $<6 \mathrm{~ms}=2 / 83$ (2.4\%)

$63(2.1 \%)$

9 cardiovascular events, including hypertension, palpitations, and tachycardia (no numbers of effect separately), 1 right bundle branch block, 1 prolonged QT interval/NR 
Table 2 continued

\begin{tabular}{|c|c|c|c|c|c|c|}
\hline Study & $\begin{array}{l}\text { Hypertension and heart rate } \\
>90 \text { th percentile }\end{array}$ & Tachycardia & Brachycardia & $\begin{array}{l}\text { Other } \\
\text { cardiovascular } \\
\text { effects }\end{array}$ & ECG abnormalities & $\begin{array}{l}\text { Discontinued } \\
\text { treatment due to } \\
\text { cardiovascular } \\
\text { effect/moment of } \\
\text { discontinuation }\end{array}$ \\
\hline $\begin{array}{l}\text { Findling } \\
\text { et al. [38] }\end{array}$ & $\begin{array}{l}33 / 265(12.5 \%) \\
\mathrm{SBP}>120 \mathrm{mmHg}+\text { increase } \\
\text { of } 10 \mathrm{mmHg} \\
4 / 265(1.5 \%) \mathrm{SBP}>140 \mathrm{mmHg} \\
20 / 265(7.5 \%) \mathrm{DBP} \\
>80-90 \mathrm{mmHg} \text { with increase } \\
\text { of } 10 \mathrm{mmHg} \\
11 / 265(4.2 \%) \\
\mathrm{HR}>100-120 \mathrm{bpm} \text {, or } \\
\text { increase of } 15 \mathrm{bpm}\end{array}$ & NR & NR & NR & $\begin{array}{l}12 / 257(4.7 \%) \\
\text { ECG HR >100 bpm }\end{array}$ & 0 \\
\hline $\begin{array}{l}\text { Wilens et al. } \\
\text { [57] }\end{array}$ & $\begin{array}{l}21 \text { increase of DBP }>10 \mathrm{mmHg} \\
5 \text { increase } \mathrm{SBP}>20 \mathrm{mmHg} \\
1 \mathrm{HR} 110-115 \mathrm{bpm} \\
6 \text { pulse change of }>25 \mathrm{bpm}\end{array}$ & 1 & NR & NR & $\begin{array}{l}34 / 138 \text { at baseline } \\
24 / 138 \text { at end point }\end{array}$ & 0 \\
\hline \multicolumn{7}{|l|}{ ATX } \\
\hline $\begin{array}{l}\text { Arcieri et al. } \\
{[43]}\end{array}$ & NR & $\begin{array}{l}6 / 350 \\
(1.7 \%)\end{array}$ & $1 / 350(0.3 \%)$ & $\begin{array}{c}1 / 350(0.3 \%) \\
\text { arrhythmia }\end{array}$ & $\begin{array}{l}\text { After } 6 \text { mo: } 8(3.6 \%) \\
\text { After } 12 \text { mo: } 7 \\
\quad(4.1 \%) \\
\text { After } 24 \text { mo: } 0 \\
\text { 1/350 }(0.3 \%) \text { a } \\
\text { lengthened QTc }\end{array}$ & $\begin{array}{l}1 \text { (arrhythmia)/ } \\
\text { after } 6 \text { mo }\end{array}$ \\
\hline $\begin{array}{l}\text { Dittmann } \\
\text { et al. [47] }\end{array}$ & $\begin{array}{l}\text { Children: } \\
\text { 13/98 (13.3\%) DBP >80 mmHg } \\
\text { 11/98 (11.2\%) } \\
\text { SBP >120 mmHg } \\
32 / 132(24.2 \%) \mathrm{HR}>100 \mathrm{bpm} \\
\text { Adolescents: } \\
6 / 34(17.6 \%) \mathrm{DBP}>80 \mathrm{mmHg} \\
3 / 34(8.8 \%) \mathrm{SBP}>130 \mathrm{mmHg} \\
16 / 34(47.1 \%)>\mathrm{SBP} \\
120 \mathrm{mmHg}\end{array}$ & NR & NR & NR & $\begin{array}{l}8 / 91(1.1 \%) \\
\mathrm{HR}>100 \mathrm{bpm} \\
\text { QTcF interval } \\
\text { change }>30 \mathrm{~ms} \\
\text { or }<60 \mathrm{~ms}=1 / 90 \\
(1.1 \%)\end{array}$ & 0 \\
\hline $\begin{array}{l}\text { Fuentes et al. } \\
\text { [40] }\end{array}$ & $\mathrm{NR}$ & NR & NR & NR & NR & 0 \\
\hline $\begin{array}{l}\text { Ghuman } \\
\text { et al. [41] }\end{array}$ & NR & NR & NR & 0 & $0 \%$ & 0 \\
\hline $\begin{array}{l}\text { Hammerness } \\
\text { et al. [42] }\end{array}$ & $1 \mathrm{HR}>120 \mathrm{bpm}$ & NR & NR & NR & $0 \%$ & 0 \\
\hline $\begin{array}{l}\text { Kratochvil } \\
\text { et al. [44] }\end{array}$ & NR & $\begin{array}{r}11 / 184 \\
(6 \%)\end{array}$ & NR & 0 & $0 \%$ & 0 \\
\hline $\begin{array}{l}\text { Sangal et al. } \\
\text { [55] }\end{array}$ & NR & NR & NR & NR & $0 \%$ & 0 \\
\hline $\begin{array}{l}\text { Yildiz et al. } \\
\text { [46] }\end{array}$ & NR & NR & NR & NR & $0 \%$ & 0 \\
\hline
\end{tabular}

$A M P$ amphetamine, $A T X$ atomoxetine, $B M I$ body mass index, $b p m$ beats per minute, $D B P$ diastolic blood pressure, $E C G$ echocardiogram, $H R$ heart rate, $M P H$ methylphenidate, $N R$ not reported, $Q T c$ QT interval corrected, $Q T c F$ QT interval corrected using Fridericia's formula, $S B P$ systolic blood pressure

Finally, $12.6 \%$ of cases reported other cardiovascular adverse events on an individual level. There were no reports of serious cardiovascular events such as myocardial infarction, stroke or sudden cardiac death, which is consistent with the literature [20, 22, 26, 27, 49]. Of note, most studies $(N=14)$ performed cardiovascular measurements 
at the screening phase, so children with cardiovascular disorders/illnesses/complaints beforehand were likely to have been excluded. This may have biased the percentage of cardiovascular effects at an individual level towards under-reporting. The vast majority of patients with other cardiovascular events continued medication treatment, with only $2 \%$ discontinuing treatment.

Our findings are consistent with prior evidence of numerically small effects of ADHD medication on changes in cardiovascular parameters (e.g. earlier reviews of clinical trials) in children, adolescents and adults with ADHD $[14,16,50]$ for most patients, although the specific pattern of effects differed to some degree. For instance, another study [14] found no effect for MPH on SBP and increased levels of BP and HR based on long-term studies in MPH (e.g. medication use for several months). An important point with cardiovascular changes over the long term is that the longer the observation period, the more likely it is that patients with clear cardiovascular effects will have dropped out. These patients will not therefore be included in the final endpoint analysis.

A prior meta-analysis on the effects of MPH and AMP on cardiovascular parameters in adult patients identified ten randomised placebo-controlled studies [17]. Medication was associated with higher mean DBP (1.0 mmHg), SBP $(2.0 \mathrm{mmHg})$ and $\mathrm{HR}(5.7 \mathrm{bpm})$. These findings suggest the effects of $\mathrm{MPH}$ on cardiovascular parameters may be somewhat larger in adults with ADHD. Notably, the studies in the adult meta-analysis do not only differ in patient age from our meta-analysis, but also in design, gender and duration. It is also difficult to directly compare mean changes of the cardiovascular parameters because paediatric norms differ from the norms for adults. Further, the adult meta-analysis included only placebo-controlled studies since 2006, the percentage of male patients was lower $(55 \%)$ and the duration of the studies was much shorter (6 weeks) than in our meta-analysis. Therefore, compared with adult ADHD populations, we should be cautious in interpreting our results, which-with MPH treatment-did not show statistically significant change of DBP and HR in children and adolescents with ADHD.

Our analyses did not find differences in adverse changes in cardiovascular parameters between the three medications studied. Analyses of possible moderators on cardiovascular pre-post changes and ADHD medication showed a statistical effect for publication year and treatment duration. Studies on MPH treatment before 2007 reported higher pre-post test changes on SBP than studies published after 2007. Furthermore, studies on AMP treatment with duration $<18$ weeks reported a higher pre-post test effect on DBP compared with longer duration studies. This is in line with our finding that the highest changes within a study of multiple measurements were reported between baseline and the first time point after, indicating a stronger shortterm effect. Unfortunately, due to lack of variation between studies with respect to age, distribution of gender, type of ADHD and comorbidity, it was not possible to explore their moderating influences. Further research is required to explore the possible effects of those moderators.

Only two studies reported cardiovascular pre-post effects of long-term medication use: one comparing MPH and ATX during 96 weeks of treatment [43], and another employing MPH for 84 weeks [35]. Both studies reported no effects on any cardiovascular parameters. It is essential to evaluate whether these overall small effects of ADHD medication on BP and HR have long-term cardiovascular consequences; for example, by inducing ventricular hypertrophy through increased cardiac load. To this end, an ongoing study named ADDUCE (Attention Deficit Hyperactivity Disorder Drugs Use Chronic Effects) has compared 24-hour blood pressure assessments and left ventricular mass measured by echocardiograms in adolescents and young adults who have been on stimulant treatment for 3 years or longer to age-matched ADHD patients who are medication-naïve (http://www.adhd-adduce.org). These data will be available for report in the near future.

\subsection{Limitations}

The results of the current meta-analysis need to be considered in the light of a number of limitations. First, 3/18 included studies did not report on cardiovascular effects at the level of the individual. Moreover, strikingly few studies on ADHD medication overall published in the international literature met inclusion criteria by providing information about cardiovascular parameters pre- and post-treatment (see Fig. 1). Second, it is usual to exclude case reports from analyses such as the one we have performed, because it is often difficult to determine causality and the results might be biased if such data were included. However, it should be acknowledged that by excluding such case reports, it is possible that a small number of individuals who have clinically significant cardiovascular adverse effects might not be drawn to the attention of clinicians; such individuals will not be obvious in the grouped mean data. Third, this meta-analysis is necessarily limited by the quality of the original reports. Fourth, nearly $80 \%$ of the studies included open-label data. Further research should utilise randomised controlled designs in children and adolescents that also focus on cardiovascular parameters and cardiovascular events. Fifth, to disentangle cardiovascular effects of medication treatment from those of the disorder itself, a control group is needed of individuals with ADHD who have never used any ADHD medication or who receive a placebo. Additionally, because of the low number of AMP studies that met our inclusion criteria, we should be 
cautious with generalizing our findings. Four AMP studies showed an overall small pre-post effect on DBP and SBP and a small to medium pre-post effect on HR. It is possible that we reported almost no effects of moderators (only duration) because of the small number (for example AMP $N=5$ ) of available studies. Most studies did not report on the timing of participants ending the study early; this has to be taken into account with the interpretation of the data. Finally, measurement issues are very important in cardiovascular studies. Few studies used adequate equipment for measurement of BP. Standard BP measurement guidelines should be followed (see [51]).

\subsection{Clinical Implications}

Medication treatment for ADHD requires ongoing cardiovascular monitoring for safety, particularly for those patients who have experienced increases in BP or HR during titration/in the short term. We recommend recording the BP and HR changes over time, especially for children and adolescents, in accordance with authoritative treatment guidelines [52, 53, NICE, 54]. Changes in BP and HR do not automatically translate into a clinically relevant cardiovascular effect such as hypertension or tachycardia. It is important that parameters are adjusted for age, BMI and gender. This should be executed at the level of the individual patient in normal treatment settings as well as in research trials as recommended by Cortese et al. [52]: "Before initiating ADHD medication, the prescribing specialist should: (1) conduct a clinical interview to detect any cardiovascular risk factor, (2) measure baseline heart rate and $\mathrm{BP}$ and (3) repeat the measure every 3 to 6 months, (4) perform an auscultation to identify any murmurs, (5) make a referral for further assessment as indicated, (6) a systematic electrocardiogram is not mandatory and should only be conducted when specifically indicated, (7) the algorithm to manage adverse cardiovascular events suggested by the European ADHD Guidelines Group [11] should be followed".

\section{Conclusion}

This meta-analysis indicates that statistically significant small to medium pre-post increases of DBP, SBP and HR were associated with AMP and ATX treatment. Furthermore, a small pre-post increase in SBP and no effect on DBP and HR were associated with MPH treatment. The clinical relevance of these mean group effects regarding the long term is unclear and requires further research, and also discussion between experts in ADHD and paediatric hypertension. These mean group effects could mask clinically relevant changes in HR and/or BP in some individuals. It is important to monitor closely the cardiovascular system in children and adolescents who are treated for ADHD with medication.

\section{Compliance with Ethical Standards}

Conflict of interest L. Hennissen and M.J. Bakker state no biomedical or financial interests or potential conflicts of interest.

T. Banaschewski has served in an advisory or consultancy role for Actelion, Hexal Pharma, Lilly, Medice, Novartis, Oxford Outcomes, PCM Scientific, Shire and Vifor Pharma. He has received conference support or speaker's fees from Medice, Novartis and Shire. He is/has been involved in clinical trials conducted by Shire and Vifor Pharma. He received royalties from Hogrefe, Kohlhammer, CIP Medien and Oxford University Press. The present work is unrelated to the above grants and relationships.

S. Carucci collaborated within projects from the European Union (7th Framework Program), has received travel support from Shire Pharmaceutical Company and has collaborated as sub-investigator in clinical trials sponsored by Shire.

D. Coghill has received research funding from Shire and the European Union, he has received consulting fees from Shire, Novartis and Sandoz and payments for lectures from Shire, Eli Lilly, Janssen Cilag and Novartis. He has given expert testimony for GlaxoSmithKline and received royalties from Oxford University Press.

M. Danckaerts has been a member of the advisory boards of Shire and Neurotech Solutions and a speaker for Shire, Medici and Novartis (no product-related lectures) over the past 3 years. Within that period she received research grants from Janssen-Cilag, Shire, EU, FWO and KU Leuven.

R.W. Dittmann is a former employee of Eli Lilly \& Co. and owner of Lilly stock. He has served in an advisory or consultancy role for Boehringer Ingelheim, Janssen-Cilag, Lilly, and Shire. He has received conference attendance support and received speaker's fees from Boehringer Ingelheim, Lilly and Shire. He has been involved in clinical trials conducted by Ferring, Janssen-Cilag, Lilly, Lundbeck, Otsuka, Servier, Shire, and Sunovion. He has received research funding from the US National Institute of Mental Health (NIMH), the European Union (EU FP7), the German Research Foundation (DFG), the German Ministries of Research and Education (BMBF) and Health (BMG/BfArM), and the Volkswagen Foundation.

C. Hollis has received research grants from the European Union FP7 programme, H2020, National Institute of Health Research (NIHR) and Medical Research Council (MRC) during the conduct of the study; $\mathrm{CH}$ is a member of the European ADHD Guideline Group (EAGG) and NICE ADHD Guideline Committee. He is not an employee of any of these companies/research organisations.

H. Kovshoff states no biomedical or financial interests or potential conflicts of interest.

S. McCarthy has, in the past 3 years, received conference attendance and research support from Shire. She has received funding from the European Union (EU FP7).

P. Nagy has served in an advisory or consultancy role for Lilly, and has received conference attendance support from Shire. He has been involved in clinical trials conducted by Shire.

E. Rosenthal received funding from the EU FP7 grant and speakers fees from Shire.

E. Sonuga-Barke has been appointed visiting chairs at Ghent University, Aarhus University and University of Sussex. He has received speaker fees, consultancy, research funding and conference support from Shire Pharma, speaker fees from Janssen Cilag, consultancy from Neurotech solutions, Aarhus University, Copenhagen University and Berhanderling, Skolerne, Copenhagen, and KU Leuven; book royalties from OUP and Jessica Kingsley; grants awarded from MRC, ESRC, Wellcome Trust, Solent NHS Trust, European 
Union, Child Health Research Foundation New Zealand, NIHR, Nuffield Foundation, Fonds Wetenschappelijk Onderzoek-Vlaanderen (FWO) and MQ-Transforming Mental Health.

I.C. K. Wong received funding from the EU FP7 grant, Hong Kong Research Grant Council and Janssen Cilag for ADHD research. He is a member of UK NICE ADHD Guidelines Development Group.

A. Zuddas has been, in the last 3 years, a consultant to/member of advisory board of/and/or speaker for Angelini, Shire, Otsuka, Takeda and Ecu Farma. He has received research funding from Shire, Roche, Lundbeck, Vifor, FP7 (PERS, ADDUCE, Stop, Matrics) and the Sardinian Regional Government, and royalties from Oxford University Press and Giunti OS.

J. K. Buitelaar has been a consultant to/member of advisory board of/ and/or speaker for Janssen Cilag BV, Eli Lilly, Bristol-Myer Squibb, Shering Plough, UCB, Shire, Lundbeck, Medice, Viforpharma, Novartis, Roche and Servier. He is not an employee of any of these companies, nor a stock shareholder of any of these companies. He has no other financial or material support, including expert testimony, patents, royalties.

Funding The research leading to these results received support from the European Community's Seventh Framework Programme (FP7/ 2007-2013) under Grant Agreement Number 260576 (ADDUCE). The Radboud University Medical Center paid the open access fee.

Open Access This article is distributed under the terms of the Creative Commons Attribution-NonCommercial 4.0 International License (http://creativecommons.org/licenses/by-nc/4.0/), which permits any noncommercial use, distribution, and reproduction in any medium, provided you give appropriate credit to the original author(s) and the source, provide a link to the Creative Commons license, and indicate if changes were made.

\section{References}

1. Polanczyk G, et al. The worldwide prevalence of ADHD: a systematic review and metaregression analysis. Am J Psychiatry. 2007;164(6):942-8.

2. Polanczyk GV, et al. Annual research review: a meta-analysis of the worldwide prevalence of mental disorders in children and adolescents. J Child Psychol Psychiatry. 2015;56(3):345-65.

3. Association, A.P. Diagnostic and statistical manual of mental disorders: DSM-5TM, 5th ed. Arlington: American Psychiatric Publishing, Inc; 2013.

4. Faraone SV, Biederman J, Mick E. The age-dependent decline of attention deficit hyperactivity disorder: a meta-analysis of followup studies. Psychol Med. 2006;36(2):159-65.

5. Banaschewski $\mathrm{T}$, et al. Long-acting medications for the hyperkinetic disorders. A systematic review and European treatment guideline. Eur Child Adolesc Psychiatry. 2006;15(8):476-95.

6. Pliszka S. Practice parameter for the assessment and treatment of children and adolescents with attention-deficit/hyperactivity disorder. J Am Acad Child Adolesc Psychiatry. 2007;46(7):894-921.

7. Taylor E, et al. European clinical guidelines for hyperkinetic disorder-first upgrade. Eur Child Adolesc Psychiatry. 2004;13(Suppl 1):I7-30.

8. (NICE), N.I.f.H.C.E. Attention Deficit Hyperactivity Disorder. Diagnosis and Management of ADHD in Children, Young People and Adults. The British Psychological Society and The Royal College of Psychiatrists; 2009.

9. (SIGN), S.I.N.G. Management of attention-deficit and hyperactivity disorders in children and young people. A National Clinical
Guideline. Scottish Intercollegial Network Guidelines (SIGN); 2009.

10. Hechtman L. Long-term treatment of children and adolescents with attention-deficit/hyperactivity disorder (ADHD). [(Hechtman) Division of Child Psychiatry, McGill University, Montreal Children's Hospital, 4018 Ste. Catherine Street West, Montreal, QC H3Z 1P2, Canada]. 2006;5:398-408. Available from: http:// ovidsp.ovid.com/ovidweb.cgi?T=JS\&PAGE=reference $\& \mathrm{D}=$ emed $7 \& N E W S=N \& A N=2006490362$.

11. Graham J, et al. European guidelines on managing adverse effects of medication for ADHD. Eur Child Adolesc Psychiatry. 2011;20(1):17-37.

12. Faraone SV, Biederman J. Neurobiology of attention-deficit hyperactivity disorder. Biol Psychiatry. 1998;44(10):951-8.

13. Volkow ND, et al. Cardiovascular effects of methylphenidate in humans are associated with increases of dopamine in brain and of epinephrine in plasma. Psychopharmacology. 2003;166(3): 264-70.

14. Stiefel G, Besag FMC. Cardiovascular effects of methylphenidate, amphetamines and atomoxetine in the treatment of attention-deficit hyperactivity disorder. Drug Saf. 2010;33(10): 821-42.

15. Hammerness PG, et al. Cardiovascular risk of stimulant treatment in pediatric attention-deficit/hyperactivity disorder: update and clinical recommendations. J Am Acad Child Adolesc Psychiatry. 2011;50(10):978-90.

16. Coghill DR, et al. A systematic review of the safety of lisdexamfetamine dimesylate. CNS Drugs. 2014;28(6):497-511.

17. Mick E, McManus DD, Goldberg RJ. Meta-analysis of increased heart rate and blood pressure associated with CNS stimulant treatment of ADHD in adults. Eur Neuropsychopharmacol. 2013;23(6):534-41.

18. Gould MS, P.D.M.P.H., et al. Sudden death and use of stimulant medications in youths. Am J Psychiatry, 2009;166(9):992-1001.

19. Johnson MRMD. Increased risk of sudden death among youths and stimulant use: determining alternate potential factors. Am J Psychiatry. 2010;167(2):214.

20. Westover AN, Halm EA. Do prescription stimulants increase the risk of adverse cardiovascular events?: a systematic review. BMC Cardiovasc Disord. 2012;12:41.

21. Hammerness PG, Surman CBH, Chilton A. Adult attention-deficit/hyperactivity disorder treatment and cardiovascular implications. Curr Psychiatry Rep. 2011;13(5):357-63.

22. Winterstein AG. Use of ADHD drugs in children and young adults does not increase risk of serious cardiovascular adverse events compared with non-use. Evid Based Mental Health. 2012;15(2):43.

23. Cooper WO, et al. ADHD drugs and serious cardiovascular events in children and young adults. $\mathrm{N}$ Engl $\mathrm{J}$ Med. 2011;365(20):1896-904.

24. Habel LA, et al. ADHD medications and risk of serious cardiovascular events in young and middle-aged adults. JAMA. 2011;306(24):2673-83.

25. Schelleman $\mathrm{H}$, et al. Cardiovascular events and death in children exposed and unexposed to ADHD agents. Pediatrics. 2011;127(6):1102-10.

26. Mazza M, et al. Drugs for attention deficit-hyperactivity disorder do not increase the mid-term risk of sudden death in children: a meta-analysis of observational studies. Int $\mathrm{J}$ Cardiol. 2013;168(4):4320-1. doi:10.1016/j.ijcard.2013.04.169 (Epub 2013 May 11).

27. Dalsgaard $\mathrm{S}$, et al. Cardiovascular safety of stimulants in children with attention-deficit/hyperactivity disorder: a nationwide prospective cohort study. J Child Adolesc Psychopharmacol. 2014;24(6):302-10. 
28. Cohen J. Statistical power analysis for the behavioral sciences (rev. ed.). Hillsdale: Lawrence Erlbaum Associates, Inc; 1977. Statistical power analysis for the behavioral sciences (rev. ed.). $\mathrm{xv}, 474$.

29. Field AP, Gillett R. How to do a meta-analysis. Br J Math Stat Psychol. 2010;63(3):665-94.

30. Kim H-W, et al. The effect of OROS methylphenidate on the sleep of children with attention-deficit/hyperactivity disorder. Int Clin Psychopharmacol. 2010;25(2):107-15.

31. Buitelaar JK, et al. Pindolol and methylphenidate in children with attention-deficit hyperactivity disorder. Clinical efficacy and side effects. Child Psychol Psychiatry Allied Discipl. 1996;37(5):587595.

32. Hammerness $P$, et al. Cardiovascular effects of longer-term, highdose OROS methylphenidate in adolescents with attention deficit hyperactivity disorder. J Pediatr. 2009;155(1):84-89.e1.

33. Lee M-S, et al. Two different solicitation methods for obtaining information on adverse events associated with methylphenidate in adolescents: a 12-week multicenter, open-label study. J Child Adolesc Psychopharmacol. 2013;23(1):22-7.

34. Wilens TE, Biederman J, Lerner M. Effects of once-daily osmotic-release methylphenidate on blood pressure and heart rate in children with attention-deficit/hyperactivity disorder results from a one-year follow-up study. J Clin Psychopharmacol. 2004;24(1):36-41.

35. Zeiner P. Body growth and cardiovascular function after extended treatment (1.75 years) with methylphenidate in boys with attention-deficit hyperactivity disorder. J Child Adolesc Psychopharmacol. 1995;5(2):129-38.

36. Coghill DR, et al. Maintenance of efficacy of lisdexamfetamine dimesylate in children and adolescents with attention-deficit/hyperactivity disorder: randomized-withdrawal study design. J Am Acad Child Adolesc Psychiatry. 2014;53(6):647-657 e1.

37. Donner R, Michaels M, Ambrosini PJ. Cardiovascular effects of mixed amphetamine salts extended release in the treatment of school-aged children with attention-deficit/hyperactivity disorder. Biol Psychiatry. 2007;61(5):706-12.

38. Findling RL, et al. A long-term open-label safety and effectiveness trial of lisdexamfetamine dimesylate in adolescents with attention-deficit/hyperactivity disorder. J Child Adolesc Psychopharmacol. 2013;23(1):11-21.

39. Wilens TE, Spencer TJ, Biederman J. Short- and long-term cardiovascular effects of mixed amphetamine salts extended-release in adolescents with ADHD. CNS Spectr. 2005;10(10 Suppl 15):22-30.

40. Fuentes $\mathrm{J}$, et al. Long-term quality-of-life and functioning comparison of atomoxetine versus other standard treatment in pediatric attention-deficit/hyperactivity disorder. J Clin Psychopharmacol. 2013;33(6):766-74.

41. Ghuman JK, et al. Prospective, naturalistic, pilot study of openlabel atomoxetine treatment in preschool children with attentiondeficit/hyperactivity disorder. J Child Adolesc Psychopharmacol. 2009;19(2):155-66.

42. Hammerness P, et al. An open study of adjunct OROS-methylphenidate in children who are atomoxetine partial responders: II. Tolerability and pharmacokinetics. J Child Adolesc Psychopharmacol. 2009;19(5):493-9.
43. Arcieri R, et al. Cardiovascular measures in children and adolescents with attention-deficit/hyperactivity disorder who are new users of methylphenidate and atomoxetine. J Child Adolesc Psychopharmacol. 2012;22(6):423-31.

44. Kratochvil CJ, et al. Atomoxetine and methylphenidate treatment in children with ADHD: a prospective, randomized, open-label trial. J Am Acad Child Adolesc Psychiatry. 2002;41(7):776-84.

45. Sangal RB, et al. Effects of atomoxetine and methylphenidate on sleep in children with ADHD. Sleep. 2006;29(12):1573-85.

46. Yildiz $\mathrm{O}$, et al. Atomoxetine and methylphenidate treatment in children with ADHD: the efficacy, tolerability and effects on executive functions. Child Psychiatry Hum Dev. 2011;42(3):25769.

47. Dittmann RW, et al. Efficacy and safety of lisdexamfetamine dimesylate and atomoxetine in the treatment of attention-deficit/ hyperactivity disorder: a head-to-head, randomized, double-blind, phase IIIb study. CNS Drugs. 2013;27(12):1081-92.

48. Hammerness $P$, et al. Cardiovascular effects of longer-term, highdose OROS methylphenidate in adolescents with attention deficit hyperactivity disorder. J Pediatr. 2009;155(1):84-9, 89 e1.

49. Olfson M, et al. Stimulants and cardiovascular events in youth with attention-deficit/hyperactivity disorder. J Am Acad Child Adolesc Psychiatry. 2012;51(2):147-56.

50. Fredriksen M, et al. Long-term efficacy and safety of treatment with stimulants and atomoxetine in adult ADHD: a review of controlled and naturalistic studies. Eur Neuropsychopharmacol. 2013;23(6):508-27.

51. O'Brien E, Asmar R, Beilin L, Imai Y, Mallion JM, Mancia G, Mengden T, Myers M, Padfield P, Palatini P, Parati G, Pickering T, Redon J, Staessen J, Stergiou G, Verdecchia P, European Society of Hypertension Working Group on Blood Pressure Monitoring. European Society of Hypertension recommendations for conventional, ambulatory and home blood pressure measurement. J Hypertens. 2003;21(5):821-848.

52. Cortese $S$, et al. Practitioner review: current best practice in the management of adverse events during treatment with ADHD medications in children and adolescents. J Child Psychol Psychiatry. 2013;54(3):227-46.

53. Taylor E, et al. European clinical guidelines for hyperkinetic disorder-first upgrade. Eur Child Adolesc Psychiatry. 2004;13(Sup 1):i7-i30.

54. Guidelines, N. Attention deficit hyperactivity disorder: diagnosis and management of ADHD in children, young people and adults. Leicester: The British Psychological Society \& The Royal College of Psychiatrists; 2009.

55. Sangal R, et al. Effects of atomoxetine and methylphenidate on sleep in children with ADHD. Sleep. 2006;29(12):1573-1585.

56. Coghill DR, et al. Maintenance of efficacy of lisdexamfetamine dimesylate in children and adolescents with attention-deficit/hyperactivity disorder: randomized-withdrawal study design. J Am Acad Child Adolescent Psychiatry. 2014;53(6):647-657.E1.

57. Wilens TE, Spencer TJ, Biederman J. Short- and long-term cardiovascular effects of mixed amphetamine salts extended-release in adolescents with ADHD. CNS Spectr. 2005;10(10, Suppl 15):22-30.

58. Ried K. Interpreting and understanding meta-analysis graphs-a practical guide. Aust Fam Physician. 2006;35(8):635-8. 